\title{
Thermodynamic, rheological and structural properties of edible oils structured with LMOGs: Influence of gelator and oil phase
}

\author{
L.H. Fasolin ${ }^{\mathrm{a}, \mathrm{b}, *}$, M.A. Cerqueira ${ }^{\mathrm{c}}$, L.M. Pastrana ${ }^{\mathrm{c}}$, A.A. Vicente ${ }^{\mathrm{b}}$, R.L. Cunha ${ }^{\mathrm{a}}$ \\ ${ }^{a}$ Department of Food Engineering, Faculty of Food Engineering, University of Campinas - UNICAMP, CEP: 13083-862, Campinas, SP, Brazil \\ ${ }^{\mathrm{b}}$ Centre of Biological Engineering, University of Minho, Campus de Gualtar, 4710-057, Braga, Portugal \\ ${ }^{\mathrm{c}}$ International Iberian Nanotechnology Laboratory, Av. Mestre José Veiga s/n, 4715-330, Braga, Portugal
}

\section{A R T I C L E I N F O}

\section{Keywords:}

Medium-chain triacylglycerol

Long-chain triacylglycerol

Crystallinity

Rheology

Organogel

Oleogel

\begin{abstract}
A B S T R A C T
The effect of different oil phases and low molecular weight organogelators (LMOGs) structures on edible oils was investigated through differential scanning calorimetry (DSC), rheology and small-angle X-ray scattering (SAXS). Different gelators (glyceryl tristearate-GT; sorbitan tristearate-ST; sorbitan monostearate-SM and glyceryl monostearate-GM) were tested in medium-chain triglycerides and high oleic sunflower (named MCT and LCT). Systems were thermoreversible and their thermodynamic properties were dependent on the combined effect of the interactions of structurants' polar head with other constituents and the sterical effect of their hydrophobic tails. The crystallization onset temperature was higher for GM and SM, possibly due to the lower sterical effect of their tails. However, the corresponding enthalpy and entropy change values were influenced by the hydrophilic head group: glycerol-based organogelator molecules were able to interact strongly than sorbitans, increasing these values. Rheological studies showed that gels produced with LCT were stronger than with MCT. Moreover, cooling and heating cycles showed more than one transition and shear dependence. Stronger structures were more sensitive to temperature, possibly because of their more organized structure that destabilizes more easily with the increase of molecular mobility. These results were in agreement with the SAXS analyses. At $50^{\circ} \mathrm{C}$, the stronger networks lost their initial structure, and at $70^{\circ} \mathrm{C}$ they collapsed. Thus, molecular interactions and structurant self-assembly were dependent on the structurant + solvent combination, leading to different physicochemical properties and thermal stability. It is expected that these results will allow customizing properties of structured oil for diverse applications, spanning from food to cosmetic and pharmaceutical industries.
\end{abstract}

\section{Introduction}

The design and modulation of the physicochemical and functional properties of supramolecular systems produced by self-assembly of their components has been one of the main areas of research in supramolecular chemistry (Balamurugan, Yeap, Mahmood, Tan, \& Cheong, 2014). Among these systems, structured oils, also called organogels and oleogels, have been considered as a new class of dynamic functional materials. They are defined as semi-solid materials resulting from the immobilization of an organic liquid in a three-dimensional network formed by a gelling or structurant agent (organogelator) that randomly entangles into fiber-like or plate-like structures (ToroVazquez et al., 2013). Structured oils can be produced using different groups of gelators: polymeric or low molecular weight organogelators (LMOGs) (Takeno, Yanagita, Motegi, \& Kondo, 2015). Polymeric gels are based on the cross-linked network of their molecules established by covalent interactions (Co \& Marangoni, 2012). For LMOGs, the driving forces responsible for supramolecular assembly are non-covalent such as H-bonding, $\pi-\pi$ stacking, solvophobic forces (hydrophobic forces for gels in water) and van der Waals interactions (George \& Weiss, 2006; Sagiri, Singh, Pal, Banerjee, \& Basak, 2015). As a result of the transient nature of the bonds, physical gels are thermo-reversible (Patel, 2017).

However, the formation, thermodynamics and physical properties, including the thermo-reversibility character of structured oils, are dependent on the molecular structure of both the oil and the gelator used and on the interactions established between them. Depending on the molecules' structure their self-assembly can assume different configurations. The gelling ability is related to the balance between the soluble and insoluble fractions of the gelator in the solvent. The gelator must be relatively insoluble to crystallize, self-assemble and form anisotropic structures. On the other hand, it must have a soluble fraction to interact with the oil moieties (Co \& Marangoni, 2012; Patel, 2017). However, the relationship between the gelator's chemical structure, its

\footnotetext{
* Corresponding author at: Department of Food Engineering, Faculty of Food Engineering, University of Campinas - UNICAMP, Campinas, SP, CEP: 13083-862, Brazil.

E-mail addresses: lh.fasolin@ceb.uminho.pt, lh.fasolin@gmail.com (L.H. Fasolin).
} 
interaction with the solvent and gelling ability is not evident $a$ priori (Mallia, George, Blair, \& Weiss, 2009; Toro-Vazquez, Morales-Rueda, Mallia, \& Weiss, 2010). Identification and understanding of the factors that govern crystal network formation and the topology of the aggregates can support the establishment of such correlation (Tang, Liu, \& Strom, 2009). This includes determining the nature of the short-range weak forces (previously mentioned) and the solubility of the gelator in the solvent, which will affect its self-assembly ability; it also includes determining thermodynamic properties and the dependence of crystal growth on the applied external forces such as shear-induced mass transfer and cooling rate (Mezzenga et al., 2005; Patel, 2017; ToroVazquez et al., 2013). These forces induce molecular alignment, network formation and define physicochemical properties of structured oils. Different structures, stability against temperature and reorganization ability will determine the applicability of a given organogel.

Sorbitans or spans can act as emulsifiers and structuring agents when solubilized in organic solvents. In particular, Span 60 and 65 show ability to produce organogels with edible oils, acting as fat replacers or delivery systems (Barbut, Wood, \& Marangoni, 2016; Kamble, Udapurkar, Nakhat, Yeole, \& Biyani, 2011; Singh, Pramanik, Ray, \& Pal, 2015). Besides, a number of studies report the use of glyceryl monostearate to produce organogels (Davidovich-Pinhas, Barbut, \& Marangoni, 2015; Lopez-Martínez, Charó-Alonso, Marangoni, \& ToroVazquez, 2015). On the other hand, glyceryl tristearate has a hydrophilic head completely esterified and it is not classified as an amphiphilic molecule. Therefore, it is usually combined with other structurant agents, but despite of this, glycerol tristearate could be used as a potential gelator of organic solvents due to its hardening properties (Cerqueira et al., 2017; Sahri \& Idris, 2010). However, each application requires a specific property, thus, it is important to understand the role of the components and the possibility to use them to fine-tune the physicochemical properties according to the product's function/specification. This work evaluated the effect of gelators with different hydrophilic heads (glyceryl and sorbitan groups) and hydrophobic tails (mono- or tri-stearic chains) and the size of oils' carbon chain (i.e. C8-C10 or C12-18) on the properties of supramolecular structures based on edible oils. We aimed at unravelling and improving knowledge about how the molecules' moieties will exert influence on the structure formed, and how these colloidal structures will change the bulk properties. The developed gelled systems were evaluated by nonisothermal oscillatory rheology, differential scanning calorimetry (DSC) and small-angle X-ray scattering (SAXS).

\section{Material and methods}

\subsection{Material}

Medium-chain triacylglycerol (MCT, Neobee 1053; composed by $55 \%$ of C8 and $44 \%$ of C10 fatty acids) was kindly donated by Stepan Lipid Nutrition (USA) and high oleic sunflower oil, used as long-chain triacylglycerol (LCT, $0.1 \%$ of C14:0, 3.8\% of C16:0, 3.3\% of C18:0, $80.1 \%$ of $\mathrm{C} 18: 1,10.7$ of $\mathrm{C} 18: 2,0.3 \%$ of $\mathrm{C} 18: 3,0.4 \%$ of $\mathrm{C} 20: 0$ and $0.1 \%$ of C20:1), was provided by Cargill (Brazil). Glyceryl tristearate (GT) (>98\%), sorbitan tristearate (ST) and sorbitan monostearate (SM) of technical grade were purchased from Sigma Aldrich Co. Ltd (USA), while glyceryl monostearate (GM) (>95.0\%) was obtained from Alfa Aesar (USA). These compounds were used as low molecular weight organogelators (LMOGs). The structure and relevant properties of the organogelators are the same as described in previous work (Cerqueira et al., 2017) and Table 1S, respectively.

\subsection{Production of supramolecular systems}

Systems were prepared in $2 \mathrm{~cm}$ diameter glass tubes with screw caps by heating the mixture of oil phase and the gelator at $80^{\circ} \mathrm{C}$ during
$30 \mathrm{~min}$ under magnetic stirring. The samples were stored at $25^{\circ} \mathrm{C} \pm 2{ }^{\circ} \mathrm{C}$ for $24 \mathrm{~h}$ to ensure the complete formation of the gel before analyses. The effect of temperature on gelled structures was evaluated for systems with 15 and $20 \%(w / w)$ gelator concentration.

\subsection{Rheological measurements}

The thermal rheological behavior of the samples was evaluated by oscillatory shear measurements in a strain-controlled rheometer Physica MCR301 (Anton Paar, Austria) equipped with a stainless-steel cone-plate geometry $\left(50 \mathrm{~mm}, 2^{\circ}\right.$ angle, truncation $\left.208 \mathrm{~mm}\right)$ in triplicate. The samples (after heating, but before cooling to $25^{\circ} \mathrm{C}$ ) were transferred onto the rheometer plate, which was preheated at $80^{\circ} \mathrm{C}$. A cooling sweep was carried out between $70{ }^{\circ} \mathrm{C}$ and $10^{\circ} \mathrm{C}$ at $5{ }^{\circ} \mathrm{C} / \mathrm{min}$, $0.1 \mathrm{~Hz}$ and $1 \%$ strain (in order to work within the linear viscoelastic region). Then a heating sweep was performed under the same conditions. The complex modulus $\left(G^{*}\right)$ and $\tan \delta\left(G^{\prime \prime} / G^{\prime}\right)$ were evaluated. The temperature of phase transition was evaluated as the point at which the slope of complex modulus vs. temperature curves changes.

\subsection{Differential scanning calorimetry (DSC)}

The crystallization and melting thermograms were determined using a differential scanning calorimeter (TA Instruments-Model 2920 Modulated DSC, New Castle, DE, USA). Supramolecular structures and pure organogelators' samples $(10 \mathrm{mg}$ ) were placed in Aluminum hermetic pans and weighted. The samples were cooled to $15^{\circ} \mathrm{C}$ and maintained at this temperature for $15 \mathrm{~min}$ before being heated to $100{ }^{\circ} \mathrm{C}$ at $5{ }^{\circ} \mathrm{C} / \mathrm{min}$. Samples were kept at this temperature for $15 \mathrm{~min}$ and then the process was repeated. These procedures (heating and cooling sets) were repeated to evaluate the gel reorganization and each sample was evaluated in triplicate. The onset temperatures $\left(T_{\text {Onset, } C}, T_{\text {Onset, } M}\right)$, peak or transition temperatures $\left(T_{C}, T_{M}\right)$ and the enthalpy changes $\left(\Delta H_{C}\right.$, $\Delta H_{M}$ ) were calculated using the Universal Analysis software (TA Instruments, New Castle, DE, USA). Subscripts $c$ and $m$ mean crystallization and melting, respectively.

The entropy change $\left(\Delta \mathrm{S}_{\mathrm{C}}\right)$ can be calculated from Eq. (1), since at the crystallization temperature the Gibb's free energy tends to zero (Lam \& Rogers, 2011; Sagiri et al., 2015).

$\Delta G=\Delta H_{C}-T_{C} \Delta S_{C}$

where $\Delta G$ and $\Delta S_{C}$ are the Gibb's free energy change and entropy change during crystallization, respectively.

\subsection{Small angle X-ray scattering (SAXS)}

Quantitative information about the temperature dependence of the gelled structures was obtained by means of small angle X-ray scattering (SAXS) measurements using the beamline of the National Synchrotron Light Laboratory (LNLS, Campinas, Brazil). A thermostatic bath was used to perform the temperature scan. The beamline was equipped with an asymmetrically cut and bent silicon monochromator $\left(\begin{array}{lll}1 & 1 & 1\end{array}\right)$ that yields a monochromatic $(\lambda=1.54 \AA)$ and horizontally focused beam. A position-sensitive X-ray detector and a multichannel analyzer were used to record the SAXS intensity, $I(q)$, as a function of the modulus of the scattering vector $q$, where $q=(4 \pi / \lambda) \sin (\theta / 2)$, with $\theta$ being the scattering angle. Each SAXS pattern corresponds to a data collection time of $100 \mathrm{~s}$.

Bragg's law was used in the determination of the crystal structure through the relative positions of the SAXS diffraction peaks. For the lamellar position peaks should obey the relationships: 1:2:3:4. The lattice parameter $d$ (lamellar periodicity) of the lamellar structures was obtained from the position $\left(s^{*}\right)$ of the first (and most intense) diffraction peak. Data analysis software ATSAS 2.7.1 was used to evaluate SAXS measurements (Petoukhov et al., 2012). 


\subsection{Statistical analyses}

All the experiments subjected to statistical analyses were carried out in triplicate. The statistical analyses were performed using analysis of variance and Tukey's mean comparison test $(p<0.05)$ using the software Statistica 10 (Statsoft Inc., USA).

\section{Results}

\subsection{Thermal behavior}

Self-sustainable structured oils were produced from 5 to $25 \%$ (w/w) organogelator; no changes of the self-assembly mechanism were observed until organogelator concentration was raised up to $15 \%(\mathrm{w} / \mathrm{w})$. At higher concentration, sterical effects became relevant, changing gel strength (Cerqueira et al., 2017). Thus, despite it is generally accepted that organogels are semi-solid materials containing low concentrations $(<15 \%)$ of organogelator (Vintiloiu \& Leroux, 2008), we decided to evaluate if this mechanism changes above that value. In addition, a high number of works has been using organogelator concentrations above $15 \%$ to produce structured oils (George \& Weiss, 2006; Matheson, Koutsos, Dalkas, Euston, \& Clegg, 2017; Moschakis, Dergiade, Lazaridou, Biliaderis, \& Katsanidis, 2017; Pénzes, Csóka, \& Eros, 2004; Sagiri et al., 2015).

Results showed that thermal events were dependent only on the organogelator since the thermal cycles for MCT-based organogels (OMCT) and LCT-based organogels (OLCT) were similar, as observed in Fig. 1. Moreover, crystallization and melting temperatures of the gels and the required energy for their formation was the same for both cooling cycles, which means that the organogels were thermoreversible (Figs. 2 and Fig. 1S). The magnitude of both $T_{\text {Onset,C }}$ and $T_{C}$ varied with the gelator in the following order: GM $>$ SM $>$ ST $>$ GT, for both types of oil. During the heating step, the highest and lowest onset melting temperatures $\left(T_{\text {Onset, } M}\right)$ were observed for GM and ST, respectively (Fig. 1S). The gelator concentration exerted influence on the thermodynamic parameters, increasing $T_{C}, T_{M}$ and both enthalpy changes. However, the difference between $T_{C}$ and $T_{M}(\Delta T)$ did not change with increasing gelator concentration, remaining close to the $\Delta T$ observed for the pure compounds, except for ST that varied with the oil used. Regarding the oil phase, the greater effect observed was in relation to the enthalpy change, since $\Delta H_{O L C T}$ was slightly higher than $\Delta H_{O M C T}$ (Table 1).

Table 1 shows the values of enthalpy and entropy changes of each thermal process. The highest values of crystallization and melting enthalpy change were observed for organogels produced with glycerolbased gelators (GM and GT). Nevertheless, $\Delta H_{M}$ was higher than $\Delta H_{C}$, since the crystals formation is not complete immediately after the crystallization thermal event and the rearrangement and strengthening of the network still occurs at lower temperatures.

The entropy change showed the same behavior of enthalpy change and structured systems based on glycerol exhibited higher values than those based on sorbitans. However, in the case of entropy change, the most important evaluation is relative to the loss of entropy in relation to the pure compound ( $\Delta S_{\text {pure }}-\Delta S_{\text {organogel }}$ ). This comparison is related to the system's organization degree and the results showed that tristearate-based organogels showed a higher loss of this property $\left(\Delta S_{\text {pure }}-\Delta S_{\text {organogel }}\right)$, which means that GT and ST are more organized and uniform gels (Table 1 ).

Rheological results showed that, in general, OLCT presented stronger gels with higher moduli than OMCT, except for organogels produced with ST, which showed similar values for both oils (Fig. 3). Moreover, the thermal scanning showed that organogels produced with monostearate organogelators (GM and SM) did not reach a plateau after gelation, which means that the structure was not completely formed. On the other hand, the tristearate-based organogels (GT and ST) presented a plateau at low temperatures (Fig. 3). The values of the complex modulus $\left(G^{*}\right)$ of organogels $15 \%(\mathrm{w} / \mathrm{w})$ showed the following order: $\mathrm{GM}>\mathrm{GT}>\mathrm{SM}>\mathrm{ST}$. However, for higher amount of gelator $(20 \% \mathrm{w} /$ w), this order presented a slight change to $\mathrm{GM} \approx \mathrm{GT} \approx \mathrm{SM}>\mathrm{ST}$. The complex modulus, $G^{*}$, reflects the contributions of elastic $\left(G^{\prime}\right)$ and viscous $\left(G^{\prime \prime}\right)$ moduli. However, for GM and ST these moduli $\left(G^{\prime}\right.$ and $\left.G^{\prime \prime}\right)$ were similar to each other, leading to values of $\tan \delta$ close to the unity. On the other hand, SM and GT presented a prevailing elastic modulus $\left(G^{\prime}\right)$ for temperatures below the sol-gel transition, with tan $\delta<1$ and a gel-like behavior (data not shown).

The rheological curves also showed more than one slope for cooling and heating sweeps (Fig. 3 and Table 2) instead of only one transition temperature as observed for DSC measurements (Fig. 2). The number of structural transitions or gel point (slopes, which are the parts of the temperature sweep curves in which $G^{*}$ increases its value) was also different depending on the organogelator and oil used. It is clear that SM and GT presented two defined slopes against only one slope observed for GM and ST, which could be related to the type of network formed, since SM and GT formed true gels $(\tan \delta<1)$. However, it is possible to observe that one of the slopes (the only one in the case of GM and the first one in the case of SM and GT) corresponds to the onset

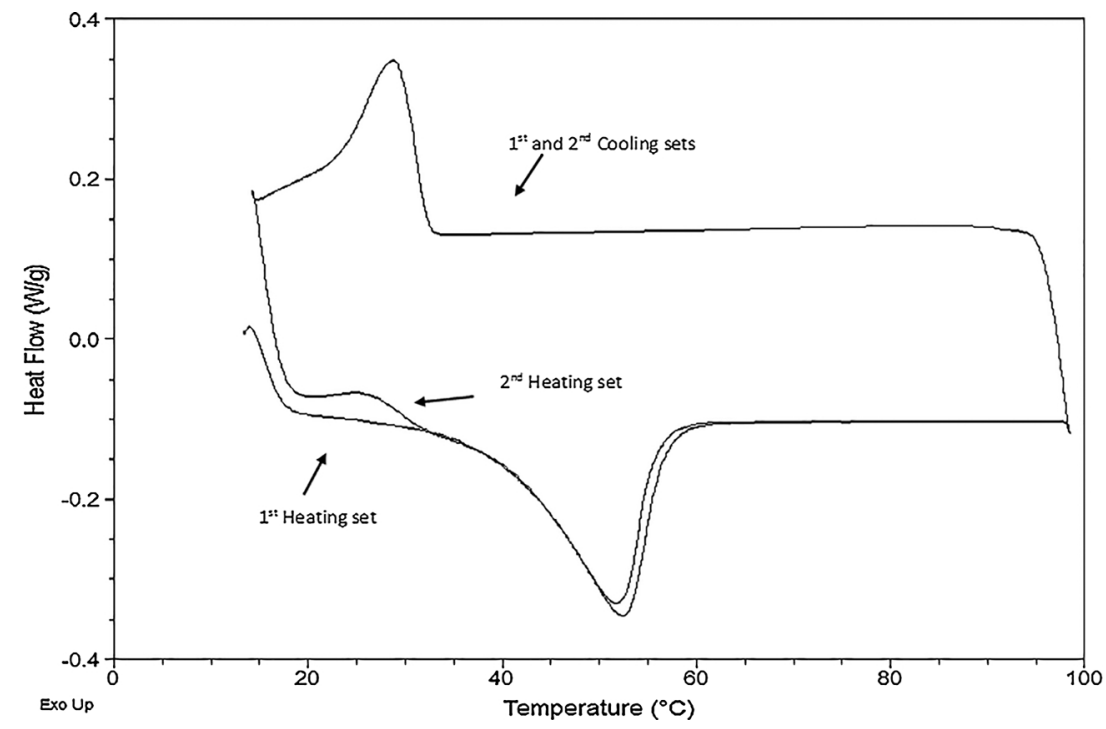

Fig. 1. Typical DSC thermogram observed for LCT organogels; the shape of the curves is also representative of thermograms obtained for MCT organogels. 

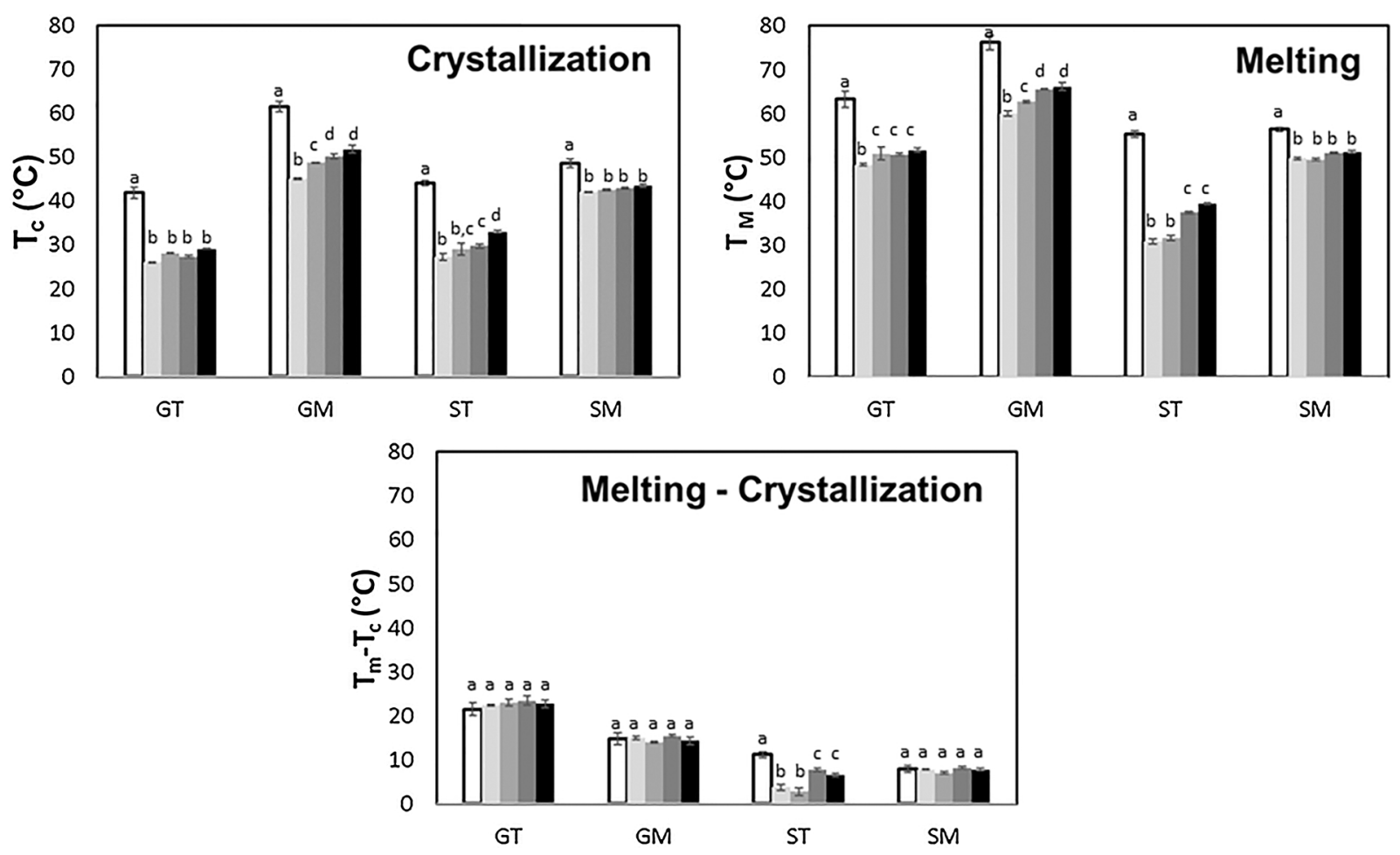

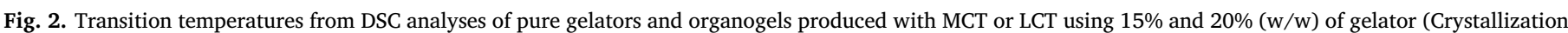
temperature $-T_{C}$; melting temperature $-T_{M}$ and their differences $\left(T_{M}-T_{C}\right)$ ). $\square$ Pure compound; MCT 15\% (w/w); $\quad$ MCT 20\% (w/w); $\square$ LCT 15\% (w/w); LCT $20 \%(w / w)$. Different letters in the same group mean a statistically significant difference $(p<0.05)$.

temperature of DSC measurements (Table 2), except for ST-based systems, in which the slope does not coincide with the temperature found in DSC measurements. In addition, some differences were observed for melting and gelation temperature depending on the type of gelator and oil used (Table 2). In general, the temperature transition of the cooling set was higher for OLCT. Moreover, the gelator concentration did not change the temperature of the slope corresponding to that of DSC measurements, that followed the sequence: ST $>$ GM $>$ SM $>$ GT for
OLCT for both concentrations ( $15 \%$ and $20 \% \mathrm{w} / \mathrm{w}$ ) and for both types of oil used. The heating set did not show significantly different temperature stability between OLCT and OMCT and depended only on the organogelator used. However, the beginning of the network melting process (first slope - Fig. 3) occurred at a lower temperature for SM and GT, showing a similar behavior to that observed in the crystallization process.

Table 1

Thermodynamic parameters for pure gelators and organogels produced with MCT (OMCT) and LCT (OLCT) using 15\% and 20\% (w/w) of gelator (melting enthalpy $\Delta H_{M}$; crystallization enthalpy $-\Delta H_{C}$; crystallization entropy $-\Delta S_{C}$ ).

\begin{tabular}{|c|c|c|c|c|c|}
\hline & Sample & $\Delta H_{M}(\mathrm{~J} / \mathrm{g})$ & $\Delta H_{C}(\mathrm{~J} / \mathrm{g})$ & $\Delta S_{C}(\mathrm{~J} / \mathrm{g})$ & $\Delta S_{\text {Pure }}-\Delta S_{\text {Organogel }}(\mathrm{J} / \mathrm{g})$ \\
\hline \multirow[t]{4}{*}{ Pure gelator } & GT & $144.8 \pm 2.24^{\mathrm{c}, *}$ & $116.8 \pm 1.77^{\mathrm{c}}$ & $2.79 \pm 0.11^{\mathrm{c}}$ & - \\
\hline & GM & $188.0 \pm 1.98^{\mathrm{d}}$ & $93.46 \pm 1.21^{\mathrm{a}}$ & $1.52 \pm 0.05^{\mathrm{a}}$ & - \\
\hline & ST & $99.72 \pm 2.00^{\mathrm{b}}$ & $90.22 \pm 1.66^{\mathrm{a}}$ & $2.05 \pm 0.07^{b}$ & - \\
\hline & SM & $73.78 \pm 1.79^{\mathrm{a}}$ & $76.97 \pm 1.45^{\mathrm{b}}$ & $1.59 \pm 0.03^{\mathrm{a}}$ & - \\
\hline \multirow[t]{8}{*}{ OMCT } & GT $15 \%$ & $24.29 \pm 0.79^{\mathrm{f}, \mathrm{A}}$ & $8.92 \pm 0.51^{\mathrm{f}, \mathrm{A}}$ & $0.34 \pm 0.02^{\mathrm{e}, \mathrm{A}}$ & $2.45 \pm 0.05^{\mathrm{d}, \mathrm{A}}$ \\
\hline & GM $15 \%$ & $26.96 \pm 1.42^{\mathrm{f}, \mathrm{B}}$ & $13.39 \pm 0.25^{\mathrm{g}, \mathrm{B}}$ & $0.30 \pm 0.01^{\mathrm{e}, \mathrm{g}, \mathrm{B}}$ & $1.22 \pm 0.02^{\mathrm{a}, \mathrm{B}}$ \\
\hline & ST $15 \%$ & $4.03 \pm 0.29^{\mathrm{e}, \mathrm{D}}$ & $3.97 \pm 0.16^{\mathrm{e}, \mathrm{D}}$ & $0.14 \pm 0.01^{\mathrm{f}, \mathrm{C}}$ & $1.91 \pm 0.03^{\mathrm{c}, \mathrm{C}}$ \\
\hline & SM $15 \%$ & $5.67 \pm 0.52^{\mathrm{e}, \mathrm{F}}$ & $1.14 \pm 0.18^{\mathrm{d}, \mathrm{F}}$ & $0.03 \pm 0.01^{\mathrm{d}, \mathrm{F}}$ & $1.56 \pm 0.03^{\mathrm{b}, \mathrm{D}}$ \\
\hline & GT $20 \%$ & $30.47 \pm 1.29^{\mathrm{g}, \mathrm{G}}$ & $7.37 \pm 1.24^{\mathrm{f}, \mathrm{H}}$ & $0.26 \pm 0.04^{\mathrm{g}, \mathrm{H}}$ & $2.53 \pm 0.06^{\mathrm{d}, \mathrm{E}}$ \\
\hline & GM $20 \%$ & $36.02 \pm 1.87^{\mathrm{h}, \mathrm{I}}$ & $17.37 \pm 2.08^{\mathrm{h}, \mathrm{J}}$ & $0.36 \pm 0.04^{\mathrm{e}, \mathrm{J}}$ & $1.16 \pm 0.04^{\mathrm{a}, \mathrm{G}}$ \\
\hline & ST $20 \%$ & $3.25 \pm 0.23^{\mathrm{e}, \mathrm{K}}$ & $2.50 \pm 0.28^{\mathrm{d}, \mathrm{e}, \mathrm{K}}$ & $0.08 \pm 0.01^{\mathrm{d}, \mathrm{f}, \mathrm{K}}$ & $1.97 \pm 0.04^{\mathrm{c}, \mathrm{H}}$ \\
\hline & SM $20 \%$ & $6.54 \pm 0.12^{\mathrm{e}, \mathrm{M}}$ & $2.29 \pm 0.33^{\mathrm{d}, \mathrm{e}, \mathrm{M}}$ & $0.05 \pm 0.01^{\mathrm{d}, \mathrm{M}}$ & $1.54 \pm 0.03^{\mathrm{b}, \mathrm{J}}$ \\
\hline \multirow[t]{8}{*}{ OLCT } & GT $15 \%$ & $25.01 \pm 0.86^{1, \mathrm{~A}}$ & $10.93 \pm 1.87^{1, \mathrm{~A}}$ & $0.40 \pm 0.07^{\mathrm{i}, \mathrm{j}, \mathrm{A}}$ & $2.39 \pm 0.06^{\mathrm{h}, \mathrm{A}}$ \\
\hline & GM $15 \%$ & $44.51 \pm 5.38^{\mathrm{k}, \mathrm{C}}$ & $16.59 \pm 1.79^{\mathrm{k}, \mathrm{C}}$ & $0.33 \pm 0.03^{\mathrm{i}, \mathrm{B}}$ & $1.19 \pm 0.03^{\mathrm{e}, \mathrm{B}}$ \\
\hline & ST $15 \%$ & $13.07 \pm 2.87^{\mathrm{j}, \mathrm{E}}$ & $5.97 \pm 0.61^{\mathrm{i}, \mathrm{E}}$ & $0.20 \pm 0.02^{\mathrm{h}, \mathrm{D}}$ & $1.85 \pm 0.04^{\mathrm{g}, \mathrm{C}}$ \\
\hline & SM $15 \%$ & $4.84 \pm 0.81^{\mathrm{i}, \mathrm{F}}$ & $2.45 \pm 0.13^{\mathrm{j}, \mathrm{G}}$ & $0.06 \pm 0.01^{\mathrm{k}, \mathrm{G}}$ & $1.53 \pm 0.02^{\mathrm{f}, \mathrm{D}}$ \\
\hline & GT $20 \%$ & $35.25 \pm 1.45^{\mathrm{m}, \mathrm{H}}$ & $13.88 \pm 1.2^{\mathrm{k}, \mathrm{I}}$ & $0.48 \pm 0.04^{\mathrm{j}, \mathrm{I}}$ & $2.31 \pm 0.05^{\mathrm{h}, \mathrm{F}}$ \\
\hline & GM $20 \%$ & $43.75 \pm 4.14^{\mathrm{k}, \mathrm{J}}$ & $21.08 \pm 1.46^{\mathrm{m}, \mathrm{J}}$ & $0.41 \pm 0.02^{\mathrm{i}, \mathrm{j}, \mathrm{J}}$ & $1.11 \pm 0.03^{\mathrm{e}, \mathrm{G}}$ \\
\hline & ST $20 \%$ & $12.47 \pm 0.65^{\mathrm{i}, \mathrm{j}, \mathrm{L}}$ & $6.19 \pm 0.41^{\mathrm{i}, \mathrm{L}}$ & $0.19 \pm 0.01^{\mathrm{h}, \mathrm{L}}$ & $1.86 \pm 0.03^{g, I}$ \\
\hline & SM $20 \%$ & $7.24 \pm 0.51^{\mathrm{i}, \mathrm{j}, \mathrm{M}}$ & $4.80 \pm 0.86^{\mathrm{i}, \mathrm{j}, \mathrm{N}}$ & $0.11 \pm 0.02^{\mathrm{h}, \mathrm{k}, \mathrm{N}}$ & $1.48 \pm 0.02^{\mathrm{f}, \mathrm{K}}$ \\
\hline
\end{tabular}

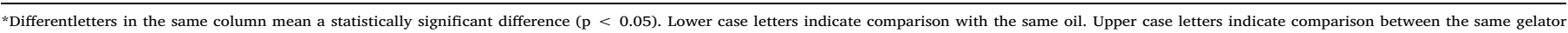
type and concentration in different oils. 

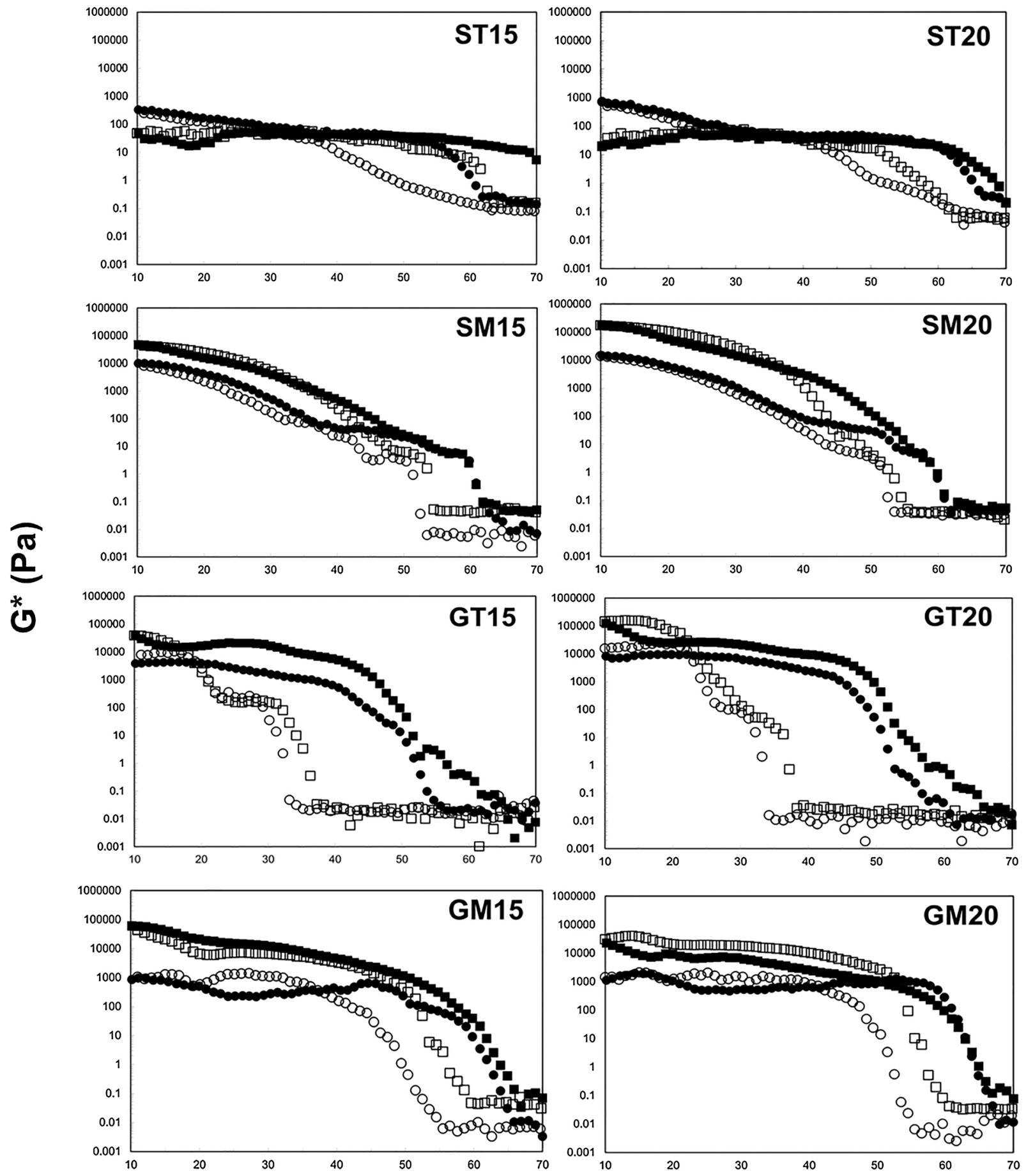

\section{Temperature $\left({ }^{\circ} \mathrm{C}\right)$}

Fig. 3. Temperature sweeps for organogels produced with MCT (๑) and LCT ( $\boldsymbol{\square})$ with $15 \%$ and $20 \%(w / w)$ of gelators. Cooling set (empty symbols) and heating set (full symbols) are shown.

\subsection{Structure evaluation}

Small-angle X-ray scattering (SAXS) spectra are characterized by sharp peaks followed by weaker reflections giving information on crystal structure. Figs. 4 shows the SAXS profiles for the systems at 25, 50 and $70{ }^{\circ} \mathrm{C}$, respectively. At $25^{\circ} \mathrm{C}$ all systems presented the formation of lamellar structures, which are characterized by the presence of one peak of higher intensity, followed by one or more peaks in the relation 1:2:3:4 (Fig. 4A). However, for OMCT produced with GM and OLCT with SM, there is the coexistence of two lamellar phases with different $d$-spacing (only the higher was shown in Table 3 ). With the temperature increase to $50^{\circ} \mathrm{C}$, the peaks were less intense but still formed lamellar structures, except in the case of some systems with SM and GT, which destabilized and did not present a defined structure (Fig. 4B). At $70{ }^{\circ} \mathrm{C}$ (Fig. 4C), all systems were destabilized with no structure formation, as a consequence of the melting of the gelators (confirmed by the absence of peaks).

The differences among the systems were related to the spacing (d) 
Table 2

Temperature transitions $\left(T_{g}\right)$ during cooling and heating sets for organogels produced with MCT and LCT using $15 \%$ or $20 \%(\mathrm{w} / \mathrm{w})$ of gelator.

\begin{tabular}{|c|c|c|c|}
\hline Oil & Sample & $T_{\text {g,cooling }}\left({ }^{\circ} \mathrm{C}\right)$ & $T_{g, \text { heating }}\left({ }^{\circ} \mathrm{C}\right)$ \\
\hline \multirow[t]{8}{*}{ MCT } & GT $15 \%$ & $25.9 \pm 1.4^{\mathrm{b}, \mathrm{A}^{*}}$ & $39.6 \pm 1.0^{\mathrm{d}, \mathrm{A}}$ \\
\hline & GT $20 \%$ & $25.1 \pm 1.8^{\mathrm{b}, \mathrm{B}}$ & $48.7 \pm 0.0^{\mathrm{a}, \mathrm{B}}$ \\
\hline & GM 15\% & $44.9 \pm 1.7^{\mathrm{a}, \mathrm{C}}$ & $47.7 \pm 1.0^{\mathrm{a}, \mathrm{D}}$ \\
\hline & GM $20 \%$ & $45.0 \pm 2.1^{\mathrm{a}, \mathrm{E}}$ & $61.9 \pm 0.0^{\mathrm{c}, \mathrm{F}}$ \\
\hline & ST $15 \%$ & $52.4 \pm 3.5^{\mathrm{c}, \mathrm{F}}$ & $58.8 \pm 1.0^{\mathrm{e}, \mathrm{H}}$ \\
\hline & ST $20 \%$ & $53.2 \pm 2.1^{\mathrm{c}, \mathrm{H}}$ & $61.9 \pm 1.0^{\mathrm{c}, \mathrm{J}}$ \\
\hline & SM $15 \%$ & $45.4 \pm 0.1^{\mathrm{a}, \mathrm{J}}$ & $51.7 \pm 0.1^{\mathrm{b}, \mathrm{K}}$ \\
\hline & SM $20 \%$ & $43.3 \pm 1.1^{\mathrm{a}, \mathrm{L}}$ & $51.7 \pm 1.1^{\mathrm{b}, \mathrm{M}}$ \\
\hline \multirow[t]{8}{*}{ LCT } & GT $15 \%$ & $27.5 \pm 1.6^{\mathrm{g}, \mathrm{A}}$ & $38.8 \pm 0.6^{\mathrm{g}, \mathrm{A}}$ \\
\hline & GT $20 \%$ & $32.2 \pm 0.1^{\mathrm{h}, \mathrm{C}}$ & $41.0 \pm 1.4^{\mathrm{f}, \mathrm{g}, \mathrm{C}}$ \\
\hline & GM $15 \%$ & $55.2 \pm 0.6^{\mathrm{e}, \mathrm{D}}$ & $61.2 \pm 1.1^{\mathrm{h}, \mathrm{E}}$ \\
\hline & GM $20 \%$ & $53.4 \pm 0.6^{\mathrm{e}, \mathrm{F}}$ & $59.2 \pm 0.7^{\mathrm{h}, \mathrm{G}}$ \\
\hline & ST $15 \%$ & $63.5 \pm 0.21^{\mathrm{f,G}}$ & $68.0 \pm 0.1^{\mathrm{i}, \mathrm{I}}$ \\
\hline & ST $20 \%$ & $64.4 \pm 2.29^{f, I}$ & $65.9 \pm 2.8^{\mathrm{i}, \mathrm{J}}$ \\
\hline & SM $15 \%$ & $43.3 \pm 1.05^{\mathrm{d}, \mathrm{K}}$ & $43.6 \pm 1.0^{\mathrm{f.L}}$ \\
\hline & SM $20 \%$ & $45.4 \pm 0.01^{\mathrm{d}, \mathrm{M}}$ & $43.6 \pm 0.58^{\mathrm{f}, \mathrm{N}}$ \\
\hline
\end{tabular}

*Differentletters in the same column mean a statistically significant difference $(p<0.05)$. Lower case letters indicate comparison with the same oil. Upper case letters indicate comparison between the same gelator type and concentration in different oils.

between the structures' units (lamella), presented in Table 3. Increasing organogelator concentration did not change the $d$-spacing, however, the type of oil chain exerted considerable effect in systems formulated with monostearate (SM and GM), which can be a consequence of the presence of lamellae with two characteristic sizes. The increase of temperature led to changes in $d$-spacing as a consequence of the network destabilization, but such effect did not show a clear trend.

\section{Discussion}

The effect of the oil chain length and organogelator structure on structured oils was evaluated through thermodynamic, physicochemical and structural properties. Results showed that depending on the domain observed (i.e., molecular, colloidal or bulk), the influence of the solvent and/or gelator was differently relevant. Thermodynamic properties showed to be dependent on the type of gelator, but they were less susceptible to the composition of the oil phase. The behavior observed for cooling and heating sets can be associated to the molecular polarity, hydrophilic-lipophilic balance (HLB), structure and solubility of the gelator and with the energies of their interactions to form the crystalline structure of the organogel. The molecules must have an insoluble portion (hydrophilic head) to induce crystal and network formation. Moreover, the hydrophobic tails need to solubilize and interact with oil moieties (Co \& Marangoni, 2012; Patel, 2017). However, the driving forces responsible for supramolecular assembly are non-covalent and short distance forces (e.g. H-bonding, dipole-dipole, donor-acceptor interactions, solvophobic forces and van der Waals interactions) (George \& Weiss, 2006; Sagiri et al., 2015), which means that the spatial/structural arrangement is also an important variable to consider (Cerqueira et al., 2017).

Crystallization temperatures $\left(T_{C}\right.$ and $\left.T_{\text {Onset, } C}\right)$ were mainly dependent on the polarity of the groups involved in these interactions and showed higher values for monostearate organogelators (GM and SM) (Fig. 2). The presence of hydroxyl groups and one hydrophobic tail has two implications: i) increase in the HLB value (Table 1S) and of the exposure of the hydrophilic head and ii) higher molecular mobility. Thus, these gelators can self-assemble more easily, decreasing the interactions between gelator and solvent, thus leading to nucleation and crystallization. However, despite of the large dipole moment of SM, glycerol is less bulky than sorbitan due to its linear disposition of $-\mathrm{OH}$ groups. This arrangement favors the approximation and interaction between the molecules, allowing them to establish H-bonds and resulting in strong attractive intermolecular forces. Moreover, for tristearate organogelators there was a synergistic effect: the higher esterification degree decreases the molecule polarity and the presence of three tails makes their molecular arrangement more rigid, thus preventing sterically the mobility of the molecules and their interactions, which displaced the crystallization process to lower temperatures.

The molecular structure also affected the energy needed (enthalpy change) to the occurrence of crystals' nucleation and network formation of organogelators (Table 1). This driving force was counterbalanced by the entropic forces associated with the phase separation/demixing of the gelator network from the homogeneous solution (Lam \& Rogers, 2011). A higher loss of entropy (entropy difference between the pure compound and the compound in solution) indicates a decrease in the crystal-solvent interfacial tension and could be related with organogels showing higher thermodynamic stability (Cao, Zhang, \& Zhang, 2010). This result is strictly related with HLB values (Table 1S) that reflect the hydrophilic and hydrophobic contributions as well as the synergistic effect between hydrophilic head and hydrophobic tails. The HLB values increase in the following order: GT $<\mathrm{ST}<\mathrm{GM}<\mathrm{SM}$, that corresponds to the inverse order of the loss of entropy (GT (2.4) $>$ ST (1.8) $>$ SM (1.5) > GM (1.2), in J/g).

The more exposed hydrophilic head group rearranges to minimize its interactions with the hydrophobic solvent. At the same time, the presence of three tails makes the gelator more soluble in the solvent, which implies that GT and ST are more organized structures with a higher degree of molecular orientation. Such higher organization degree was reflected in a more uniform structure, with one-size lamellae occurring for GT and ST, in comparison to the mixture of lamellae sizes observed for GM and SM (Fig. 4A). These results can be related with enthalpy change results. The nucleation of glycerol-based organogelators (GM and GT) was more exothermic in comparison to that of sorbitans. For GM, this result is related to the higher interaction of the molecule's polar groups and H-bond formation. The less bulky spatial arrangement of GM allows the approximation of the moieties and their stronger interaction, since hydrophobic interactions, the dipole moment and $\mathrm{H}$-bonding are short-range weak forces. However, for GT, not only the polar group but also the alkyl chain shows relevance on the molecular interactions. A discussion in the same direction was done in other works where gelators with amides and carboxyl groups were used, showing that the type of interactions and their relevance is dependent on the gelator's structure (Abraham et al., 2012; Sato, Yoshimoto, Suzuki, Kobayashi, \& Kaneko, 1990; Toro-Vazquez et al., 2013). This can also be applied to ST organogels, which presented higher values of $\Delta H_{C}$ than SM.

Rheology results showed that the magnitude of $G^{*}$ depends on the molecular characteristics of the gelator and on its concentration (Fig. 3). The presence of glycerol hydrophilic heads led to stronger gels than those obtained with sorbitans. As mentioned above, glycerol is smaller and less bulky in comparison to sorbitans, being able to interact more closely with its neighbors, which lead to gels with stronger elastic structures and resistance to deformation. Moreover, gels produced with monostearate tended to be stronger than those produced with tristearate (Fig. 3).

At this point it is important to reinforce that higher organization and thermodynamic stability does not mean necessarily stronger gels. The self-assembly of the gelators is dependent on multivariable factors, as mentioned above, which will exert influence not only at the molecular but also at the colloidal and bulky levels. At these levels, the oil phase started to exert a more significant influence on structural and physicochemical properties. SAXS measurements showed that the oil type changed the $d$-spacing (Table 3), which can be related with rheology results (Table 2). Generally, rheological results showed that gels produced with LCT were stronger than OMCT, showing higher $\mathrm{G}^{*}$ values.

Analyzing the results from the heating-set, the most relevant information was obtained from structural and rheological measurements. DSC showed that, in the same way as for the cooling set, the structure stability against temperature was also dependent on the synergy 


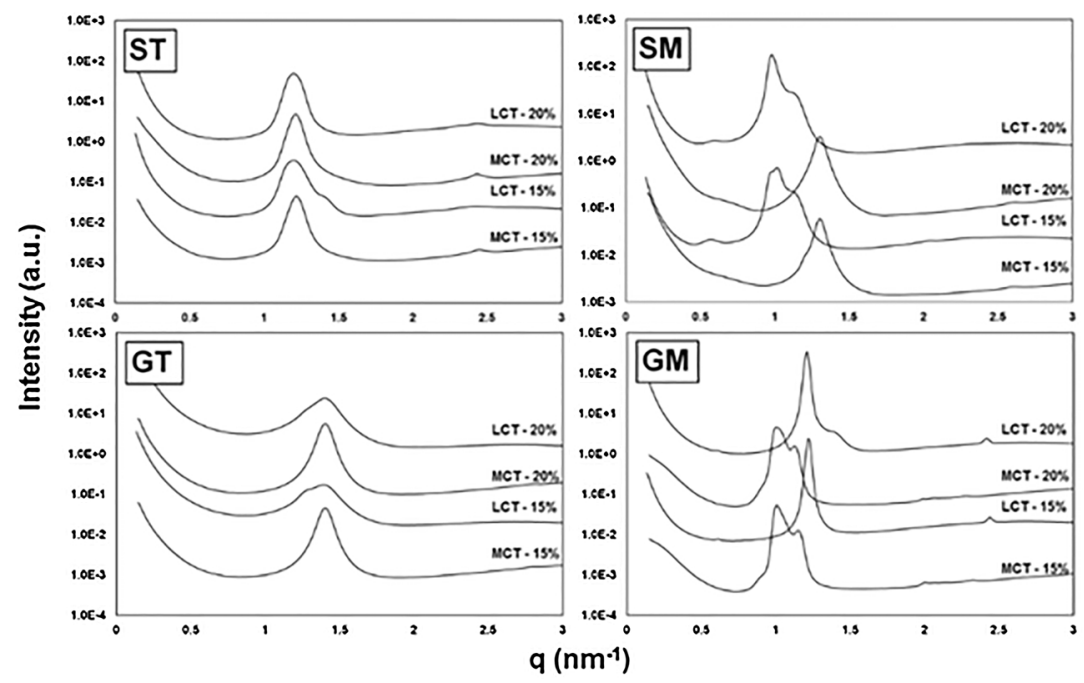

A
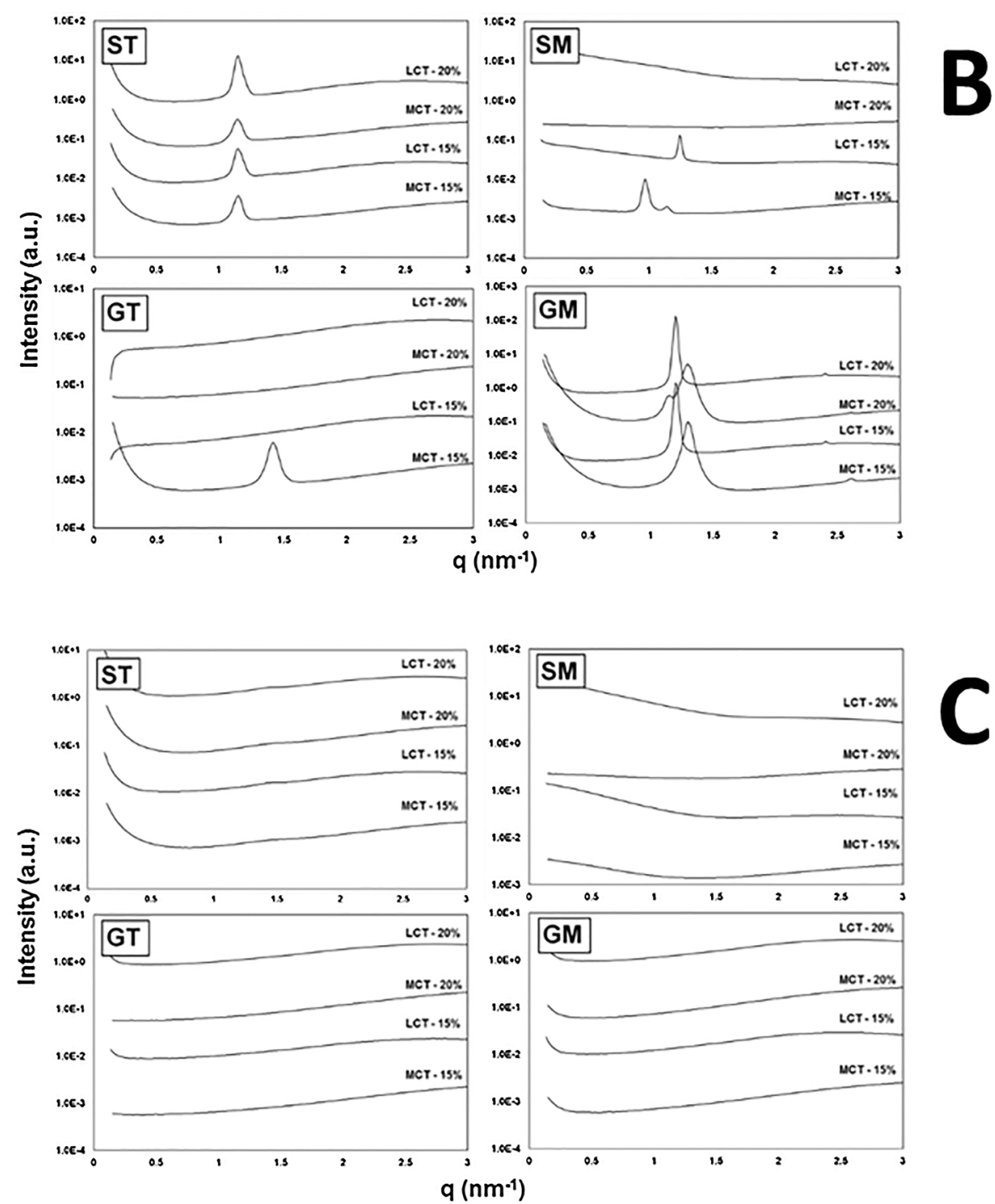

Fig. 4. SAXS patterns for structured oils formulated with MCT and LCT at different temperatures: A) $25^{\circ} \mathrm{C}$; B) $50{ }^{\circ} \mathrm{C}$ and $\mathrm{C}$ ) $70{ }^{\circ} \mathrm{C}$. 
Table 3

Values of $d$-spacing ( $\mathrm{nm}$ ) evaluated by SAXS at different temperatures for organogels produced with MCT and LCT with $15 \%$ and $20 \%(\mathrm{w} / \mathrm{w})$ of organogelators. Values in nanometers $(\mathrm{nm})$.

\begin{tabular}{|c|c|c|c|c|c|c|c|}
\hline \multicolumn{2}{|c|}{ Gelator (w/w) } & \multicolumn{6}{|c|}{ Temperature } \\
\hline & & \multicolumn{2}{|l|}{$25^{\circ} \mathrm{C}$} & \multicolumn{2}{|l|}{$50{ }^{\circ} \mathrm{C}$} & \multicolumn{2}{|l|}{$70^{\circ} \mathrm{C}$} \\
\hline & & MCT & LCT & MCT & LCT & MCT & LCT \\
\hline \multirow[t]{2}{*}{ GT } & $15 \%$ & 4.47 & 4.49 & 4.43 & $\mathrm{~N}$ & $\mathrm{~N}$ & $\mathrm{~N}$ \\
\hline & $20 \%$ & 4.47 & 4.46 & $\mathrm{~N}$ & $\mathrm{~N}$ & $\mathrm{~N}$ & $\mathrm{~N}$ \\
\hline \multirow[t]{2}{*}{ GM } & $15 \%$ & 6.46 & 5.15 & 4.98 & 5.22 & $\mathrm{~N}$ & $\mathrm{~N}$ \\
\hline & $20 \%$ & 6.50 & 5.21 & 5.10 & 5.23 & $\mathrm{~N}$ & $\mathrm{~N}$ \\
\hline \multirow[t]{2}{*}{ ST } & $15 \%$ & 5.15 & 5.26 & 5.41 & 5.43 & $\mathrm{~N}$ & $\mathrm{~N}$ \\
\hline & $20 \%$ & 5.17 & 5.24 & 5.46 & 5.43 & $\mathrm{~N}$ & $\mathrm{~N}$ \\
\hline \multirow[t]{2}{*}{ SM } & $15 \%$ & 4.82 & 6.26 & 5.47 & 5.02 & $\mathrm{~N}$ & $\mathrm{~N}$ \\
\hline & $20 \%$ & 4.81 & 6.40 & $\mathrm{~N}$ & $\mathrm{~N}$ & $\mathrm{~N}$ & $\mathrm{~N}$ \\
\hline
\end{tabular}

${ }^{*} \mathrm{~N}=$ no structure observed.

between the interactions of the hydrophilic groups and the sterical effects, once the hysteresis between $T_{M}$ and $T_{C}$ in the organogels was similar to that of the pure compounds (Fig. 2). This difference is related to the strength of the interactions and consequently to the energy needed to break them. The stronger polar group interaction (GM) showed a high value of $T_{\text {Onset, } M}$, while sorbitan head with three tails (ST) showed the lowest values of that variable (Fig. 1S). However, rheology showed that when the gels were heated $G^{*}$ started to decrease, which can be related to the reduction of the entanglements in the gelator network, mostly due to hydrophobic interactions, that are the weakest forces established between molecules (Fig. 3). Thus, when the temperature reaches the gel-sol transition a sudden decrease in $G^{*}$ was observed, indicating the breakdown of the gelator network and the collapse of the structure (Pernetti, van Malssen, Kalnin, \& Flöter, 2007). This process can be observed in the number of different slopes present in the heat-set that varied with the oil-gelator combination, since gelsol transition is not a simple first-order process but takes place within a certain temperature range and can be related to the breakdown of different types of interactions (Laupheimer, Preisig, \& Stubenrauch, 2015) (Fig. 3).

\section{Conclusion}

Structured oils were produced with medium-chain and long-chain triacylglycerols using gelators with different structures. The influence of the structure of solvent and organogelator was different, depending on the level studied (molecular, colloidal or macroscopic). However, thermodynamic properties (molecular level), influenced on the structure formed (colloidal level) and changed the rheological properties (macroscopic level). Moreover, the organogelator self-assembly and its ability to form the organogel network was dependent of multivariable factors, such as molecular polarity, HLB, conformational structure and solubility in the solvent. These factors exerted effect on the type, strength and relevance of the interactions between the molecular groups. Monostearates are less bulky gelators with linear structure, which increase the molecular mobility and allow the higher organization of the surfactant. Moreover, the glycerol polar heads are more prone to interact with each other in comparison to sorbitans, producing stronger gels. On the other hand, the presence of three alkyl chains acted synergistically with the polar heads in building a stronger structure (reflected in an increased gel strength). This happens due to their entanglement and to the hydrophobic interactions established between them. Finally, the oil phase also changed the physicochemical properties and stronger gels with higher complex moduli were obtained with long-chain triacylglycerols. Thus, gelator self-assembly and organogel strength were dependent of synergistic effects between molecular interactions and spatial arrangement (sterical effects). Moreover, the results suggest that it is possible to produce materials with different properties, suitable for diverse industrial applications. Both hard and soft gels can be used as vehicles for hydrophobic bioactives, as fat replacers or texturizer agents in cosmetic, pharmaceutical, medical and food products. Physicochemical properties, thermal stability and reversibility can be tuned, combining oil phases and organogelators with different properties, to obtain a product with the appropriate characteristics.

\section{Acknowledgements}

Luiz H. Fasolin thanks the scholarship Capes/FCT program (349/13) for the research exchange and Rosiane Lopes Cunha thanks CNPq (CNPq 307168/2016-6) for the productivity grant. The authors also would like to thank Fapesp (EMU 09/54137-1), CNPq and Capes for their financial support and the Brazilian Synchrotron Light Laboratory (LNLS) for the opportunity to carry out SAXS measurements. This study was also supported by the Portuguese Foundation for Science and Technology (FCT) under the scope of the strategic funding of UID/BIO/ 04469/2013 unit and COMPETE 2020 (POCI-01-0145-FEDER-006684) and BioTecNorte operation (NORTE-01-0145-FEDER-000004) funded by the European Regional Development Fund under the scope of Norte2020 - Programa Operacional Regional do Norte. and of the Project RECI/BBB-EBI/0179/2012 (FCOMP-01-0124-FEDER-027462).

\section{Appendix A. Supplementary data}

Supplementary data associated with this article can be found, in the online version, at https://doi.org/10.1016/j.foostr.2018.03.003.

\section{References}

Abraham, S., Lan, Y., Lam, R. S. H., Grahame, D. A. S., Kim, J. J. H., Weiss, R. G., \& Rogers, M. A. (2012). Influence of positional isomers on the macroscale and nanoscale architectures of aggregates of racemic hydroxyoctadecanoic acids in their molecular gel, dispersion, and solid states. Langmuir, 28(11), 4955-4964. http://dx. doi.org/10.1021/la204412t.

Balamurugan, S., Yeap, G. Y., Mahmood, W. A. K., Tan, P. L., \& Cheong, K. Y. (2014). Thermal and photo reversible gel-sol transition of azobenzene based liquid crystalline organogel. Journal of Photochemistry and Photobiology A: Chemistry, 278, 19-24. http://dx.doi.org/10.1016/j.jphotochem.2013.12.013.

Barbut, S., Wood, J., \& Marangoni, A. (2016). Potential use of organogels to replace animal fat in comminuted meat products. Meat Science, 122, 155-162. http://dx.doi. org/10.1016/j.meatsci.2016.08.003.

Cao, S., Zhang, Y., \& Zhang, Y. (2010). Nucleation and morphology of monosodium aluminate hydrate from concentrated sodium aluminate solutions. Crystal Growth and Design, 10(4), 1605-1610.

Cerqueira, M. A., Fasolin, L. H., Picone, C. S. F., Pastrana, L. M., Cunha, R. L., \& Vicente, A. A. (2017). Structural and mechanical properties of organogels: Role of oil and gelator molecular structure. Food Research International, 96, 161-170. http://dx.doi. org/10.1016/j.foodres.2017.03.021.

Co, E. D., \& Marangoni, A. G. (2012). Organogels: An alternative edible oil-structuring method. JAOCS, Journal of the American Oil Chemists' Society, 89(5), 749-780. http:// dx.doi.org/10.1007/s11746-012-2049-3.

Davidovich-Pinhas, M., Barbut, S., \& Marangoni, A. G. (2015). The role of surfactants on ethylcellulose oleogel structure and mechanical properties. Carbohydrate Polymers, 127, 355-362. http://dx.doi.org/10.1016/j.carbpol.2015.03.085.

George, M., \& Weiss, R. G. (2006). Molecular organogels. Soft matter comprised of lowmolecular-mass organic gelators and organic liquids. Accounts of Chemical Research, 39(8), 489-497. http://dx.doi.org/10.1021/ar0500923.

Kamble, S. R., Udapurkar, P., Nakhat, P. D., Yeole, P. G., \& Biyani, K. R. (2011). Development and evaluation of sorbitan monostearate organogels as a topical delivery system for aceclofenac. Indian Journal of Pharmaceutical Education and Research, 45(1), 65-70.

Lam, R. S. H., \& Rogers, M. A. (2011). Activation energy of crystallization for trihydroxystearin, stearic acid, and 12-hydroxystearic acid under nonisothermal cooling conditions. Crystal Growth and Design, 11(8), 3593-3599. http://dx.doi.org/10.1021/ cg200553t.

Laupheimer, M., Preisig, N., \& Stubenrauch, C. (2015). The molecular organogel n-decane/12-hydroxyoctadecanoic acid: Sol-gel transition, rheology, and microstructure. Colloids and Surfaces A: Physicochemical and Engineering Aspects, 469, 315-325. http:// dx.doi.org/10.1016/j.colsurfa.2015.01.039.

Lopez-Martínez, A., Charó-Alonso, M. A., Marangoni, A. G., \& Toro-Vazquez, J. F. (2015) Monoglyceride organogels developed in vegetable oil with and without ethylcellulose. Food Research International, 72, 37-46. http://dx.doi.org/10.1016/j.foodres. 2015.03.019. 
Mallia, V. A., George, M., Blair, D. L., \& Weiss, R. G. (2009). Robust organogels from nitrogen-containing derivatives of (R)-12-hydroxystearic acid as gelators: Comparisons with gels from stearic acid derivatives. Langmuir, 25(15), 8615-8625. http://dx.doi.org/10.1021/la8042439.

Matheson, A. B., Koutsos, V., Dalkas, G., Euston, S., \& Clegg, P. (2017). Microstructure of $\beta$-Sitosterol: $\gamma$-Oryzanol edible organogels. Langmuir, 33(18), 4537-4542. http://dx. doi.org/10.1021/acs.langmuir.7b00040.

Mezzenga, R., Meyer, C., Servais, C., Romoscanu, A. I., Sagalowicz, L., \& Hayward, R. C. (2005). Shear rheology of lyotropic liquid crystals: A case study. Langmuir, 21(8), 3322-3333. http://dx.doi.org/10.1021/la046964b.

Moschakis, T., Dergiade, I., Lazaridou, A., Biliaderis, C. G., \& Katsanidis, E. (2017). Modulating the physical state and functionality of phytosterols by emulsification and organogel formation: Application in a model yogurt system. Journal of Functional Foods, 33, 386-395. http://dx.doi.org/10.1016/j.jff.2017.04.007.

Pénzes, T., Csóka, I., \& Eros, I. (2004). Rheological analysis of the structural properties effecting the percutaneous absorption and stability in pharmaceutical organogels. Rheologica Acta, 43(5), 457-463. http://dx.doi.org/10.1007/s00397-004-0396-1.

Patel, A. R. (2017). A colloidal gel perspective for understanding oleogelation. Current Opinion in Food Science, 15, 1-7. http://dx.doi.org/10.1016/j.cofs.2017.02.013.

Pernetti, M., van Malssen, K., Kalnin, D., \& Flöter, E. (2007). Structuring edible oil with lecithin and sorbitan tri-stearate. Food Hydrocolloids, 21(5-6), 855-861. http://dx. doi.org/10.1016/j.foodhyd.2006.10.023.

Petoukhov, M. V., Franke, D., Shkumatov, A. V., Tria, G., Kikhney, A. G., Gajda, M., ... Svergun, D. I. (2012). New developments in the ATSAS program package for smallangle scattering data analysis. Journal of Applied Crystallography, 45(2), 342-350. http://dx.doi.org/10.1107/S0021889812007662.

Sagiri, S. S., Singh, V. K., Pal, K., Banerjee, I., \& Basak, P. (2015). Stearic acid based oleogels: A study on the molecular, thermal and mechanical properties. Materials Science and Engineering C, 48, 688-699. http://dx.doi.org/10.1016/j.msec.2014.12.
018.

Sahri, M. M., \& Idris, N. A. (2010). Palm stearin as low trans hard stock for margarine. Sains Malaysiana, 39(5), 821-827.

Sato, K., Yoshimoto, N., Suzuki, M., Kobayashi, M., \& Kaneko, F. (1990). Structure and transformation in polymorphism of petroselinic acid. Journal of Physical Chemistry, 94(18), 3180-3185. http://dx.doi.org/10.1021/j100370a078.

Singh, V. K., Pramanik, K., Ray, S. S., \& Pal, K. (2015). Development and characterization of sorbitan monostearate and sesame oil-based organogels for topical delivery of antimicrobials. AAPS PharmSciTech, 16(2), 293-305. http://dx.doi.org/10.1208/ s12249-014-0223-7.

Takeno, H., Yanagita, M., Motegi, Y., \& Kondo, S. (2015). Relationship between helical aggregates and polymorphs in a 12-hydroxystearic acid gel: Their thermal stability and formation kinetics. Colloid and Polymer Science, 293(1), 199-207.

Tang, S., Liu, X. Y., \& Strom, C. S. (2009). Producing supramolecular functional materials based on fiber network reconstruction. Advanced Functional Materials, 19(14), 2252-2259.

Toro-Vazquez, J. F., Morales-Rueda, J., Mallia, V. A., \& Weiss, R. G. (2010). Relationship between molecular structure and thermo-mechanical properties of candelilla wax and amides derived from (R)-12-hydroxystearic acid as gelators of safflower oil. Food Biophysics, 5(3), 193-202. http://dx.doi.org/10.1007/s11483-010-9159-y.

Toro-Vazquez, J. F., Morales-Rueda, J., Torres-Martínez, A., Charó-Alonso, M. A., Mallia, V. A., \& Weiss, R. G. (2013). Cooling rate effects on the microstructure, solid content, and rheological properties of organogels of amides derived from stearic and (R)-12hydroxystearic acid in vegetable oil. Langmuir, 29(25), 7642-7654. http://dx.doi. org/10.1021/la400809a.

Vintiloiu, A., \& Leroux, J.-C. (2008). Organogels and their use in drug delivery - A review. Journal of Controlled Release, 125(3), 179-192. http://dx.doi.org/10.1016/j.jconrel. 2007.09.014. 